\title{
A CANNABIS SATIVA E SUAS PROPRIEDADES FARMACOLÓGICAS NO TRATAMENTO DE TRANSTORNO DE ANSIEDADE - REVISÃO SISTEMÁTICA
}

\author{
Karoliny Mascarenhas Carvalho ${ }^{1}$ \\ Layane dos Santos Souza ${ }^{2}$ \\ Paloma Lima da Silva ${ }^{3}$ \\ Sâmia Paula Santos Neves Oliveira ${ }^{4}$
}

RESUMO: Introdução: A fisiopatologia do transtorno de ansiedade envolve sistemas, estruturas e neurotransmissores, que são alterados de acordo fatores ambientais, podendo haver inúmeros mecanismos de ação. As pesquisas demográficas hoje indicam uma perspectiva em que a ansiedade abrange uma área de evidências entre as principais crises psicológicas, estando à frente da depressão. Segundo dados da Organização Mundial da Saúde (OMS), o Brasil tem o maior índice de pessoas ansiosas do mundo: I8,6 milhões de brasileiros (9,3\% da população) convivem com o transtorno. A Cannabis sativa, (planta popularmente conhecida como maconha), é composta por mais de 400 substâncias dentre as quais se destacam o tetrahidrocanabinol (THC) e o canabidiol (CBD) pelos seus benefícios na ansiedade. Objetivo: Apresentar os efeitos do CBD e THC conforme resultados associados com a ansiedade em animais de laboratório e humanos. Metodologia: Realizou-se uma revisão de literatura, com levantamento na base de dados PudMed, SciElo, ScienceDirect e Google acadêmico, dos estudos experimentais que testaram a combinação de CBD e THC nas condições subredito foi realizado. Resultado: Dos estudos localizados na base de dados, I9 atenderam aos critérios de inclusão, com a relação do sistema endocanabinóide e com a fisiopatologia do transtorno de ansiedade, foram evidenciadas respostas promissoras com o uso de CBD e THC, obtendo-se resultados ansiolíticos, conforme a segurança quanto ao uso. Conclusão: Diante disso, há evidências que a Cannabis sativa, principalmente o CBD, pode ser uma terapia alternativa para o controle da ansiedade por apresentar uma menor recorrência de danos colaterais em comparação aos medicamentos usualmente utilizados.

Palavras-chaves: Ansiedade. Cannabis Canabidiol.

\footnotetext{
${ }^{\mathrm{I}}$ Bacharelado Em Farmácia pela Universidade Salvador.

². Bacharelado Em Farmácia pela Universidade Salvador. E-mail: layanessantos@live.com

${ }^{3}$ Bacharelado Em Farmácia pela Universidade Salvador.

${ }^{4}$ Orientadora do curso de Farmácia da Universidade Salvador Bacharelado Em Farmácia.
} 
ABSTRACT: Introduction: The pathophysiology of anxiety disorder involves systems, structures, and neurotransmitters, which are altered according to environmental factors, and there may be numerous mechanisms of action. Demographic surveys today indicate a view that anxiety encompasses an area of evidence among the major psychological crises, leading to depression. According to data from the World Health Organization (WHO), Brazil has the highest rate of anxious people in the world: 18.6 million Brazilians (9.3\% of the population) live with the disorder. Cannabis sativa, (a plant popularly known as marijuana), is composed of more than 400 substances among which tetrahydrocannabinol (THC) and cannabidiol (CBD) stand out for their benefits in anxiety. Objective: To present the effects of CBD and THC as results associated with anxiety in laboratory animals and humans. Method: A literature review was carried out, with a survey in the PudMed, SciElo, ScienceDirect and Academic Google database, of the experimental studies that tested the combination of CBD and THC in underreporting conditions was carried out. Results: Of the studies located in the database, I9 met the inclusion criteria, with the relationship of the endocannabinoid system and the pathophysiology of the anxiety disorder, promising responses were evidenced with the use of CBD and THC, obtaining anxiolytic results, according to safety regarding use. Conclusion: Therefore, there is evidence that Cannabis sativa, especially $\mathrm{CBD}$, can be an alternative therapy for anxiety control as it presents a lower recurrence of collateral damage compared to commonly used medications.

Keywords: Anxiety. Cannabis Cannabidiol.

\section{INTRODUÇÃO}

A ansiedade é considerada a doença do século XX1. A Organização Mundial de Saúde (OMS) relata que nos últimos is anos o aumento da incidência desse transtorno em todo o mundo foi significativo, com o Brasil no topo da lista (PATAH, 2019). As pesquisas demográficas hoje indicam uma perspectiva em que a ansiedade abrange uma área de evidências entre as principais crises psicológicas, estando à frente da depressão. Conforme a base de dados da Organização Mundial da Saúde (OMS), o Brasil tem a maior relação de pessoas ansiosas do mundo: 18,6 milhões de brasileiros (9,3\% da população) que convivem com o transtorno.

O transtorno de ansiedade na Classificação de Transtornos Mentais e de Comportamento da CID-io, como transtornos ansiosos ( $\left.\mathrm{F}_{4} \mathrm{I}\right)$. 
$\mathrm{F}_{40}$ - Transtornos fóbico-ansiosos

- F40.o Agorafobia - fobias relacionadas ao medo de locais públicos ou de viajar sozinho, por exemplo.

I. F40.I Fobias sociais - medo de ser exposto.

2. F40.2 Fobias específicas (isoladas) - fobia a situação específica como a proximidade de alguns animais ou espaço fechado.

3. F40.8 Outros transtornos fóbico-ansiosos.

4. F40.9 Transtorno fóbico ansioso não especificado.

F4II - Ansiedade generalizada.

5. F4I.o Transtorno de pânico (ansiedade paroxística episódica) - ataques de pânico.

6. F4I.I Ansiedade generalizada - não ocorre exclusivamente de modo preferencial numa determinada situação, os sintomas são variáveis.

F41.2 - Transtorno misto ansioso e depressivo - o paciente apresenta ao mesmo tempo sintomas ansiosos e depressivos.

F41.3 - Outros transtornos ansiosos mistos - características de outros transtornos.

F418 - Outros transtornos ansiosos especificados - Histeria de angústia.

F419 - Transtorno ansioso não especificado.

O transtorno de ansiedade é uma condição desagradável de tensão, apreensão inquietação, medo, sendo estes os sintomas leves, na maioria das situações de origem 
desconhecida (KEYLA CRYSTINA). Os sintomas graves são similares aos do medo, porém com taquicardia, sudoração, tremores e palpitações. Os transtornos mentais envolvendo a ansiedade são os mais comuns.

Esses sintomas somáticos e psíquicos juntos dependendo do grau pode causar um transtorno à vida, alterando a rotina do paciente. Episódios de ansiedade leve são fatores da rotina diária que não evidencia o tratamento com fármacos. Contudo, os sintomas intensos, podem ser tratados com óleo essencial, ansiolíticos, antidepressivos, benzodiazepínicos de ação mais longa, como (clonazepam, lorazepam e diazepam). Embora não sejam indicados contra certos quadros de ansiedade.

A expectativa do uso do canabidiol (CBD) como opção terapêutica para transtorno de ansiedade deve-se aos efeitos psicoativos e na cognição, qualidade, segurança nos ensaios clínicos com resultados positivos e o amplo espectro de ações farmacológicas. O efeito terapêutico para transtorno de ansiedade é reconhecido na erva. Contudo, o uso abusivo pode apresentar consequências adversas, tais como taquicardia, secura da boca e olhos avermelhados (ALVES, 2020). A Canabis sativa é utilizada para tratamentos multimodais. Podendo ser usado para tratar vários prognósticos e coexistentes, portanto, com potencial de ajudar a reduzir a carga da polifarmácia (MACALLUM; RUSSO; 2018)

Pesquisas admitem que existam canabinóides endógenos e que seus receptores, no sistema nervoso central (SNC) sejam alvos do canabidiol resultante da Cannabis sativa. Recentemente, a Agência Nacional de Vigilância Sanitária (ANVISA) e o Conselho Federal de Medicina - CFM autorizaram o uso do canabidiol para fins terapêuticos (RESOLUÇÃO CFM № 2.113 / 2014), contudo este uso se restringe aos pacientes com epilepsia refratária em tratamento. Até o presente, as pesquisas estão restritas a experimentos com animais e estudos clínicos com humanos em fase I e II (SILVA; REIS; SANTOS; ABEU; SANTOS; DOURADO; RESENDE; 2017). 
Diante disso, o objetivo deste estudo é analisar estudos relacionados à Cannabis sativa, para fins terapêuticos no tratamento do transtorno de ansiedade no Brasil, especificamente entre os anos de 2017 e 2021. A questão referente à utilização do canabidiol e tetraidrocanabinol na conjuntura da farmacologia brasileira é vigente e à amplificação do tema tem impulsionado à sociedade científica a estimular as discussões que envolvem os aspectos éticos, médicos sociais sobre adesão aos medicamentos compostos à base de Cannabis sativa (planta popularmente conhecida como maconha) (VIEIRA; MARQUES; SOUSA, 2020).

\section{MATERIAL E MÉTODO}

A pesquisa foi descritiva e qualitativa, com a elaboração de a uma revisão sistemática, que buscou encontrar na planta Cannabis sativa a função farmacológica como um ansiolítico para fim medicinal tendo como fonte principal o tratamento para transtorno de ansiedade, baseando-se no conhecimento da farmacologia da planta.

Para tanto, recorreu-se à busca de artigos científicos originais e de revisão nas bases de dados eletrônicas como PudMed, SciElo, ScienceDirect e Google acadêmico. Cabe evidenciar que, inicialmente foram lidos os resumos e a metodologia desses artigos e em seguida, foi feita a leitura completa dos textos.

Os critérios de inclusão dos artigos levantados foram: publicados entre os anos de 2017 e 202I, em língua inglesa, portuguesa e espanhola, selecionados pelas metodologias experimentais definida e padronizada, farmacocinética e farmacodinâmica. A pesquisa gerada é qualitativa e os dados dos artigos compilados são multidisciplinares, permeando as áreas de farmacognosia, farmacocinética e farmacodinâmica. A FIGURA I lista o número de estudos encontrados nas pesquisas e quantos deles foram incluídos e excluídos com base nos critérios adotados. 
Figura I - Desenvolvimento de triagem dos estudos incluídos na revisão sistemática.
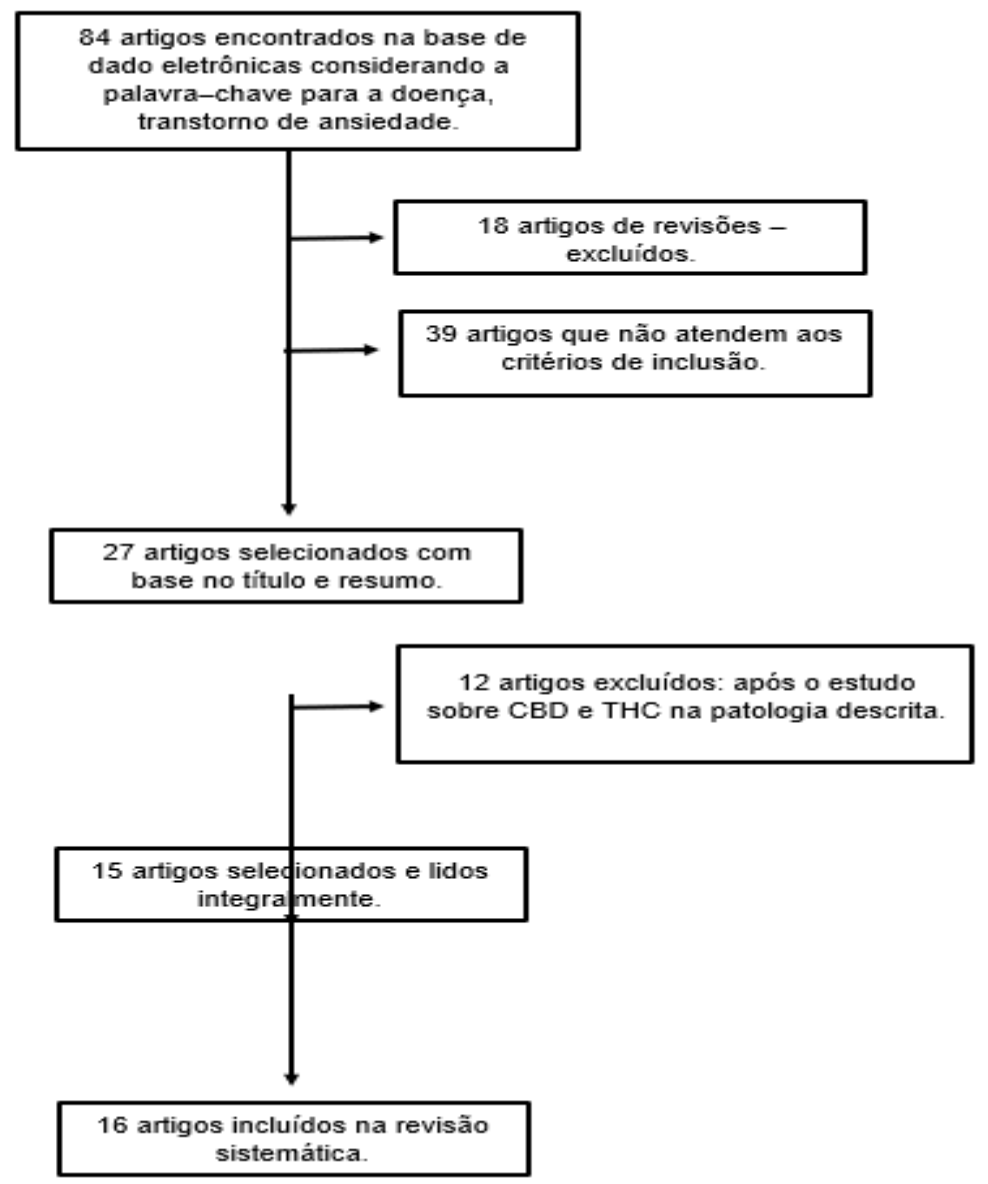

\section{RESULTADO E DISCUSSÃO}

Os 15 estudos listados entre os anos de 2017 e 2021 serão apontados e abordados a seguir, visando demonstrar o status atual da utilização da Cannabis sativa para fim medicinal no uso específico de transtorno de ansiedade. Os resultados obtidos encontram-se apresentados na TABELA I, organizado conforme os números, autores, títulos e anos de publicação. Conforme ilustrado na Figura I, dos 84 artigos localizados 
pela pesquisa inicial, 15 artigos atenderam a revisão sistemática. Tendo em vista assegurar a sensibilidade aos resultados do estudo e permitir sua reprodutibilidade. 
Revista Ibero- Americana de Humanidades, Ciências e Educação- REASE

Tabela I: Relação de artigos científicos listados e examinados na pesquisa de revisão integrativa.

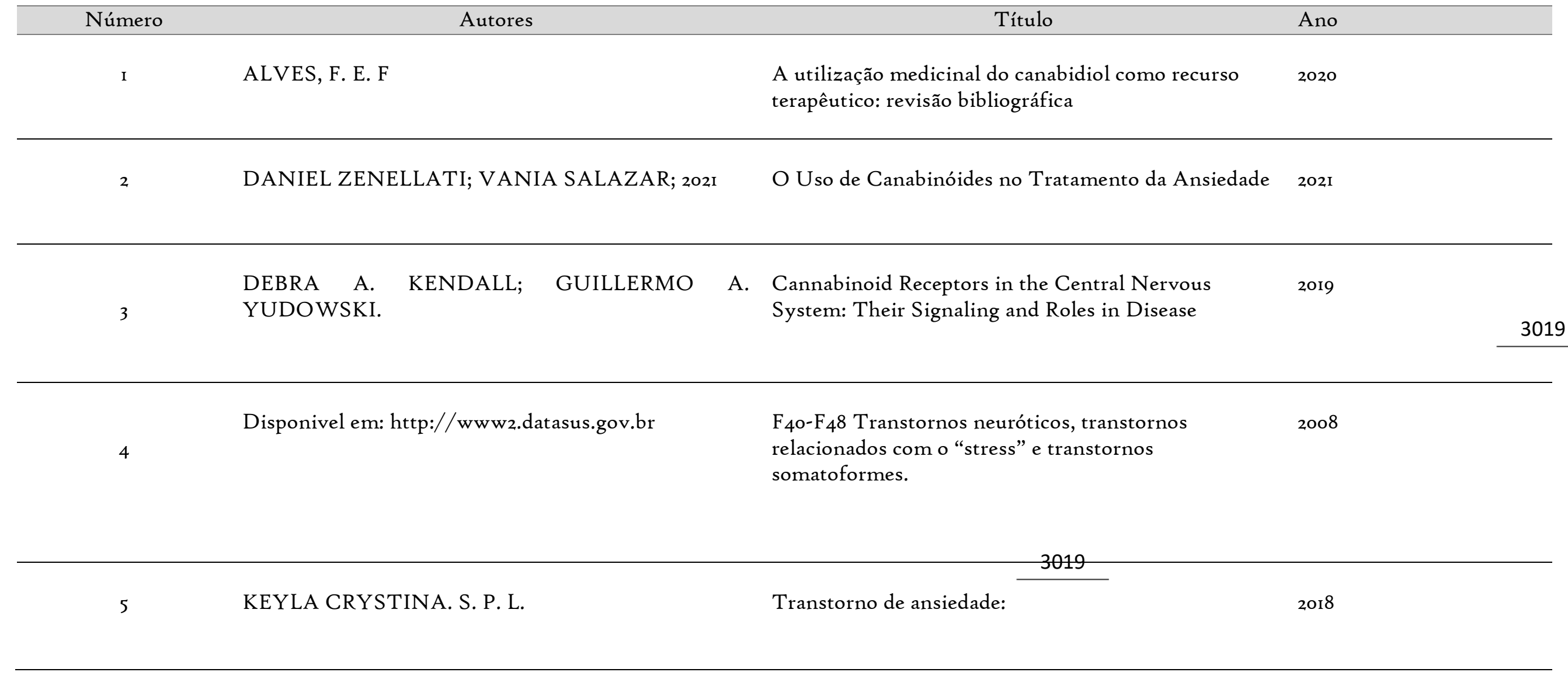


LUANA, DOS S. F P; IASMIN F. M. L;

CAROLINA. P. S; LUANA. G. P, VANESSA. B. S.

R.L; KÁSSIA REGINA. S; FRANCISCO. B. P. J:
Ansiedade: o uso da Cannabis sativa como terapêutica 2020

alternativa frente aos benzodiazepínicos

(1)

(1)

.




\begin{tabular}{|c|c|c|c|c|}
\hline 13 & SARAH V. V & $\begin{array}{l}\text { Evidências Científicas da Associação de Canabidiol e } \\
\text { Tetrahidrocanabinol na Ansiedade, na Depressão e na } \\
\text { Esquizofrenia }\end{array}$ & 2019 & 3021 \\
\hline I4 & $\begin{array}{l}\text { SILVA, D. O. F, REIS. M. C, SANTOS. B. E. M, } \\
\text { ABEU. C. F, SANTOS. L. V. C, DOURADO, M. S. } \\
\text { C. I, RESENDE. V. V. L, }\end{array}$ & $\begin{array}{l}\text { O Uso do Canabidiol no Tratamento da Ansiedade } \\
\text { The Use of Cannabidiolin the Treatment of Anxiety, } \\
\qquad 3021\end{array}$ & 2017 & \\
\hline 15 & VIEIRA, S. L & $\begin{array}{l}\text { O uso de Cannabis sativa para fins terapêuticos no } \\
\text { Brasil: uma revisão de literatura: Scientia Nauralis. }\end{array}$ & 2020 & \\
\hline
\end{tabular}


A partir das pesquisas sobre transtorno de ansiedade selecionados, as subdivisões abrangeram: experimentos em humanos e animais de laboratório, as proporções de CBD e THC calcularam e avaliaram se a proporção testada foi eficaz nas respostas à ansiedade, (SARAH VIGNOTO, 2019).

À vista disso, a metodologia abordada por meio dos estudos dos modelos experimentais em animais, tem como objetivo detectar farmacocinética, farmacodinâmica e a estudos comportamentais gerais entre os animais. Tais testes comportamentais representam importante contribuição ligada a execução nos períodos de avaliação, considerando respostas condicionadas quanto incondicionadas. Neste trabalho de revisão, foi usado um método de avaliação, sendo a tabela de testes, referindo-se ao processo de análise das pesquisas de artigos científicos referentes a ansiedade, no entanto, para melhor análise, conferir apêndice, ao final da dissertação. Segue abaixo a Tabela 2 com a documentos dos estudos levantados, seguindo-se à diante com a descrição dos resultados. Nota-se que o CBD e o THC foram testados em variadas proporções, em diferentes espécies de animais de laboratório, em humanos; algumas dessas proporções se mostraram, inclusive eficazes (referindo as o5 quantidades de experimentos de CBD e THC), que associados promoveram efeito moderador da resposta examinada, para a ansiedade. Em contraste, algumas proporções não demonstraram diferença, por exemplo quando comparadas ao placebo. 
Revista Ibero- Americana de Humanidades, Ciências e Educação- REASE ${ }_{\text {open }}^{\text {access }}$

Tabela 2 - Listagem bibliográfico de estudos experimentais em humanos e animais com canabinóides (CBD, THC) de 2017 a 2019

\begin{tabular}{|c|c|c|c|c|}
\hline Canabinóide & Dose & $\begin{array}{l}\text { Modelo } \\
\text { experimental }\end{array}$ & Objetivo & Referência \\
\hline CBD e THC & 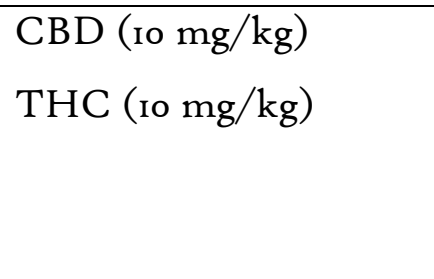 & $\begin{array}{l}\text { camundongos } \\
\text { machos }\end{array}$ & $\begin{array}{l}\text { Analisar a hiperatividade locomotora de rebote que foi } \\
\text { inibida parcialmente pelo CBD. O CBD não alterou a } \\
\text { ansiedade persistente induzida pelo THC e nem a } \\
\text { tolerância aos efeitos farmacológicos do THC }\end{array}$ & 2017 \\
\hline CBD & 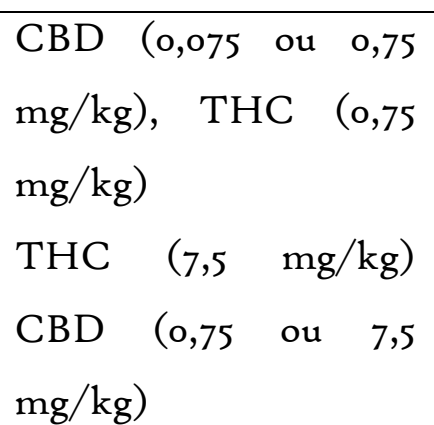 & $\begin{array}{l}\text { ratos Wistar } \\
\text { machos e fêmeas }\end{array}$ & $\begin{array}{l}\text { CBD teria efeitos mínimos em termos de tolerabilidade } \\
3023\end{array}$ & 2018 \\
\hline
\end{tabular}


Revista Ibero- Americana de Humanidades, Ciências e Educação- REASE

\begin{tabular}{|c|c|c|c|c|}
\hline $\begin{array}{l}\text { CBD } \\
\text { sintético }\end{array}$ & 6oomg & $\begin{array}{l}32 \text { pacientes de alta } \\
\text { paranoia }\end{array}$ & $\begin{array}{l}\text { Investigar efeitos de uma única dose de CBD sintético } \\
\text { sobre o pensamento paranoico e ansioso }\end{array}$ & 2018 \\
\hline $\begin{array}{l}\text { CBD, THC, } \\
\mathrm{CBD}+\mathrm{THC}\end{array}$ & Doses variadas & $\begin{array}{lr}\text { Ratos machos com } \\
\text { ansiedade } & \text { e } \\
\text { depressão } & \end{array}$ & $\begin{array}{l}\text { Investigar avaliar os efeitos do CBD, THC, CBD + } \\
\text { THC em ratos }\end{array}$ & 2019 \\
\hline $\mathrm{CBD}$ & 40omg & $\begin{array}{lr}\text { Io homens } & \text { com } \\
\text { transtorno } & \text { de } \\
\text { ansiedade } & \\
\text { generalizado } & \end{array}$ & Investigar as bases neurofisiológicas dos efeitos do CBD & 2011 \\
\hline
\end{tabular}


A tabela acima indica resultados de dois artigos selecionados sobre a combinação de CBD e THC em humanos e animais. Foram testados o CBD e THC em diferentes proporções de dosagem, metodologias, tempo de avaliação pósadministração, foco de avaliação, qualidade, número de doses administradas, uso agudo ou crônico e via de administração. Algumas dessas se mostraram eficazes e outras não, diante de cada critério de estudo. Entre os diferentes autores foram observados que o CBD bloqueia os efeitos do THC tanto nos animais, quanto nos humanos (DANIEL ZENELLATI; VANIA SALAZAR; 202I).

Existem muitas divergências quanto a natureza dos efeitos de canabinóides, o autor, como Daniel Zenellati cita o THC como ansiogênico, enquanto o autor Arnold apresenta o CBD oscilando os efeitos psicomimético do THC, potencializando seus efeitos positivos. Esse balanceamento dos pontos positivos e negativos é conhecido como efeito comitiva, sendo obtido pela utilização conjunta dos canabinóides, teoricamente apresentando melhor eficácia terapêutica. Pacientes psiquiátricos paranoico e ansioso, cujo exame dos efeitos de uma única dose de CBD, não foi capaz de reduzir a ansiedade e não teve efeito sobre ocorrências paranoides.

Buscar as bases neurofisiológicas dos efeitos do CBD na diminuição da ansiedade, facilitando a habituação de ansiedade na fobia social. A administração de CBD diminuiu a ansiedade rapidamente e de forma sustentada. No entanto, aumentou a ansiedade e não teve efeitos na construção de resultados positivos. Em contraste, os estudos com pacientes com transtorno de ansiedade social foram notórios a que o CBD não apresentou o efeito ansiolítico em voluntários saudáveis com traços paranoides elevados. (MARÍA S; FRANCISCO N; ANI G; AMAYA A; FRANCISCO S; 2019)

Os estudos com animais permitiram entender melhor alguns aspectos a respeito da utilização do canabinóides, como: relação entre doses de THC e CBD, ausência de efeitos adversos com utilização de CBD em tipos diferentes de ansiedade, de respostas do CBD em espécies, bem como, o efeito ansiolítico e antidepressivo de THC com posologias diferentes. Esses dados ajudam a dar suporte 
para realizar novos estudos e compreender melhor os resultados obtidos em humanos (DANIEL ZENELLATI; VANIA SALAZAR 202I).

O sistema canabinóides é dividido em três tipos: endocanabinóide, fitocanabinóides e canabinóides sintéticos. Endocanabinóide são mensageiros produzidos pelo nosso organismo; Fitocanabinóides, substâncias lipofílicas da planta (THC, CBD, CBN), o CBD e CBN são capazes de produzir efeitos medicinais, pois são derivados do THC. Os canabinóides exógenos e endógenos se ligam a receptores específicos, como: $\mathrm{CB}$ i e $\mathrm{CB} 2$. $\mathrm{O}$ CBI é mais aparente no sistema nervoso central e o $\mathrm{CB}_{2}$ no sistema imunológico e hematopoiético (DANIEL ZENELLATI; VANIA SALAZAR 2021).

Os canabinoides deve-se ao $\Delta 9$-tetra-hidrocanabinol (THC), sendo o princípio ativo principal da Cannabis juntamente com os receptores canabinoides específicos, denominados receptores $\mathrm{CB}_{1}$ e $\mathrm{CB}_{2}$ e de ligantes endógenos (endocanabinoides), ambos são receptores acoplados à proteína G. Dentro dos sistemas nervosos centrais, o $\mathrm{CB}$ I está primariamente localizado nos terminais nervosos pré-sinápticos e é responsável pela maioria dos efeitos neurocomportamentais dos canabinoides juntamente com os mecanismos relativos à síntese e eliminação. $\mathrm{O}_{\mathrm{CB}}$ ao contrário, é o principal receptor de canabinoide no sistema imune, mas também pode manifestar-se nos neurônios. Os principais agonistas endógenos de $\mathrm{CB}_{1}$ e $\mathrm{CB}_{2}$ são os derivados do ácido araquidônico (RANG et al., 2016).

$O$ receptor $\mathrm{CB}_{\mathrm{I}}$ são encontrados no cérebro, sendo um neurotransmissor central, tem efeito psicotrópico, distribuídas em partículas que provoca uma recompensa psicológica ao estriado e córtex central, hipotálamo, cerebelo, sendo mais concentrados no hipocampo. A ativação do receptor $\mathrm{CB}$ I se dá a hiperpolarização dos neurônios e há uma diminuição da liberação dos neurotransmissores. (RANG et al., 2016). Os receptores do $C B_{I}$ nos neurônios pós-sinápticos regulam a atração e a plasticidade simpática via modulação dos canais de potássio, inibição da adenilciclase com ativação do MAPK e inibição da PKA, dessa forma, ao ligar-se o receptor CBı, o 
THC interfere nos efeitos do SNC (DEBRA A. KENDALL; GUILLERMO A. YUDOWSKI, et al SARAH VIGNOTO, 2019).

$\mathrm{O}$ receptor $\mathrm{CB}_{2}$ possui apenas cerca de $45 \%$ de homologia de aminoácidos, localizado principalmente no tecido linfoide. Os agonistas $\mathrm{CB}_{2}$ possuem efeitos antiateroscleróticos. À vista disso, os receptores $\mathrm{CB}_{2}$ têm potentes efeitos antiinflamatórios e neurotransmissores modulando a liberação de citocinas (RANG et al., 2016).

A tabela de publicações cientificas, constatou que o número de publicações envolvendo o estudo com a Cannabis sativa em todo o mundo é menor do que de fato poderia existir. Sendo perceptível nos experimentos selecionados para essa revisão, que não houve muitos estudos com humanos, supõe-se que as leis proibicionistas estejam delimitando as pesquisas práticas com a planta e seus derivados. Em síntese, nota-se que o uso da Cannabis adotada no âmbito terapêutico não é uma novidade na medicina. Talvez essa inversão de prioridades se dê por conta dos impostos, questões governamentais, já que os investimentos em pesquisas são baixos.

\section{CONCLUSÃO}

Há evidências científicas de que o CBD é capaz de atenuar respostas associadas à ansiedade induzidas pelo THC em animais de laboratório e humanos. Com base nos resultados analisados, a proporção de CBD:THC com possível finalidade terapêutica, ou seja, uma que contém CBD em concentração igual ou maior do que a do THC, é a mais testada e eficaz nos estudos analisados.

A partir dos artigos examinados, há evidências da atuação do canabinóides em regiões cerebrais especificas envolvidas na ansiedade, ocorrendo redução da ansiedade em níveis fisiológicos. O efeito ansiolítico ocorreu em modelos saudáveis e com transtornos ansiosos, além do mais, foi mostrado a segurança quanto ao uso dos canabinóides utilizados no presente estudo.

Sendo perceptível nos experimentos selecionados para essa revisão, que não houve muitos estudos com humanos. Foram observadas limitações que podem impactar na 
qualidade da evidência, tais como reduzidos tamanhos amostrais e heterogeneidade da dose de escolha. Por outro lado, observa-se a adoção de desfechos primários relevantes na prática clínica, que estabiliza do grau de ansiedade.

Em síntese, nota-se que o uso da Cannabis adotada no âmbito terapêutico não é uma novidade na medicina. Foram observadas limitações que podem impactar na qualidade da evidência, tais como reduzidos tamanhos amostral e heterogeneidade da dose de escolha. Por outro lado, observa-se a adoção de desfechos primários relevantes na prática clínica, com escolha de instrumentos validados para determinação do grau de ansiedade.

\section{REFERENCIAS}

ALVES, F. E. F. A utilização medicinal do canabidiol como recurso terapêutico: revisão bibliográfica, The medicinal use of cannabidiol as therapeutic resource: review. Aceito em: 15.04.2020.

ANDRADE, J. V. et al. Ansiedade, um dos problemas do século XXI. Revista de Saúde Reages, Paripiranga, v. 2, n. 4, p. 34-39, jun. 2019.

CONRADO. S. M. M. Centro Terapêutico para tratamento de depressão e ansiedade

https://repositorio.animaeducacao.com.br/bitstream/ANIMA/I4342/3/CONRAD

ODOSSANTOSMARQUESMOTTA_monografia.pdf2020

DANIEL. Z; VANIA CRISTINA. R S. O Uso de Canabinóides no Tratamento da Ansiedade. https://rbmc.emnuvens.com.br/rbmc/article/view/97

DEBRA A. KENDAll; GUILlERMO A. YUDOWSKI. Cannabinoid Receptors in the Central Nervous System: Their Signaling and Roles in Disease 
F40-F48 Transtornos neuróticos, transtornos relacionados com o "stress" e transtornos somatoformes. Disponivel em: http://www2.datasus.gov.br/cidio/V20o8/WebHelp/f4o_f $48 . h t m$.

KEYLA CRYSTINA. S. P. L. Transtorno de ansiedade: https://revistasfacesa.senaaires.com.br/index.php/iniciacaocientifica/article/view/47/14. 2018

LUANA, DOS S. F P; IASMIN F. M. L; CAROLINA. P. S; LUANA. G. P, VANESSA. B. S. R.L; KÁSSIA REGINA. S; FRANCISCO. B. P. J: Ansiedade: o uso da Cannabis sativa como terapêutica alternativa frente aos benzodiazepínicos file://C:/Users/Lenovo/Downloads/13797-35773-I-PB.pdf

MACALLUM, C. A; RUSSO, E. B, Practical considerations in medical cannabis administration and dosing, https://doi.org/ı.ı.16/j.ejim.2018.oı.004

MARÍA S; FRANCISCO N; ANI G; AMAYA A; FRANCISCO S. Cannabidiol: A Potential New Alternative for the Treatment of Anxiety, Depression, and Psychotic Disorders file://C:/Users/Lenovo/Downloads/biomolecules-10-or575v2.pdf

ORGANIZAÇÃO MUNDIAL DA SAÚDE. DEPRESSÃO, 2017. Disponível em: http://bvsms.saude.gov.br/ultimas-noticias/2408-o7-4-dia-mundial-da-saude-2017depressao. Acesso em: or jun. 202I.

ORGANIZAÇÃO MUNDIAL DA SAÚDE. ANSIEDADE Disponível em: https:exame.com/ciencia/brasil-e-o-pais-mais-ansioso-do-mundo-segundo-aoms/2019

PATAH, Rodrigo. Ansiedade: reflexões sobre o mal do século XXI-2019

RANG, H. P. et al. Farmacologia. 8. ed. Rio de Janeiro: Elsevier, 2016. .

SARAH V. V: Evidências Científicas da Associação de Canabidiol e Tetrahidrocanabinol na Ansiedade, na Depressão e na Esquizofrenia 
https://repositorio.ufsc.br/bitstream/handle/123456789/214413/PFMC-Poo27-

D.pdf? sequence $=-\mathrm{I} \&$ isAllowed $=\mathrm{y}$

SILVA, D. O. F, REIS. M. C, SANTOS. B. E. M, ABEU. C. F, SANTOS. L. V. C, DOURADO, M. S. C. I, RESENDE. V. V. L, O Uso do Canabidiol no Tratamento da Ansiedade The Use of Cannabidiolin the Treatment of Anxiety, publicado em $28 / 05 / 2017$

VIEIRA, S.L et al. $\mathrm{O}$ uso de Cannabis sativa para fins terapêuticos no Brasil: uma revisão de literatura: Scientia Nauralis: SCIENTIA NAURALIS, Paraíba, v. 2, n. 2, p. 901-919, 24 ago. 2020.

VIVIANE M. S; CARSTEN T. W; FABRÍCIO A. M: Exploração farmacológica do sistema endocanabinoide: novas perspectivas para o tratamento de transtornos de ansiedade e depressão?

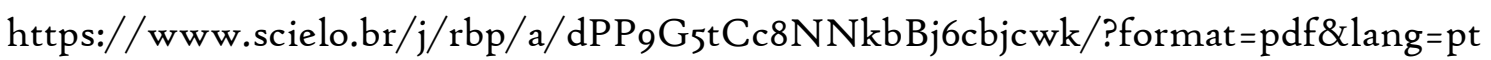

